\title{
neuroRAD 2019
}

\section{Keynote-Speaker - Grundkurs Künstliche Intelligenz - StAR-Lounge - Power-Pitch-Vorträge - Pepper zu Gast in Frankfurt}

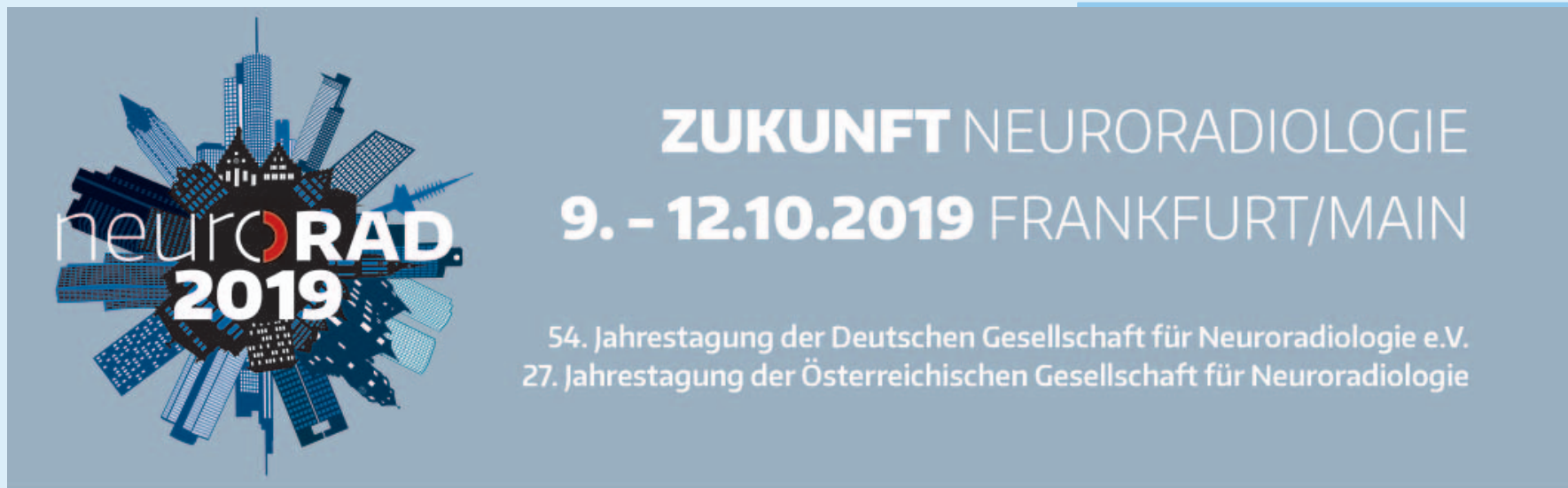

Die 54. Jahrestagung der Deutschen Gesellschaft für Neuroradiologie und gleichzeitig die 27. Jahrestagung der Österreichischen Gesellschaft für Neuroradiologie findet in $\mathbf{2 0 1 9}$ vom 9. bis 12. Oktober im Kap Europa in Frankfurt am Main unter dem Motto „Zukunft Neuroradiologie“ statt. Längst der Höhepunkt des neuroradiologischen Kongressjahres, wartet der diesjährige „neuroRAD“ mit Altbewährtem, aber auch zahlreichen Neuerungen auf.

\section{Von Berlin über Maastricht bis nach Calgary - die KEYNOTE-SPEAKER 2019}

Vierzehn Keynote-Speaker aus dem In- und Ausland sprechen auf dem neuroRAD 2019 über spannende Themen aus der Neuroradiologie und schauen auch über den Tellerrand hinaus. Lesen Sie im Folgenden, auf wen Sie sich im Herbst in Frankfurt freuen dürfen.

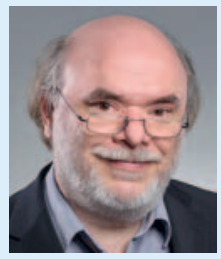

\section{Prof. Philipp Slusallek}

ist wissenschaftlicher Direktor am Deutschen Forschungszentrum für Künstliche Intelligenz (DFKI) in Saarbrücken. Vor seiner Tätigkeit am DFKI war Prof. Slusallek Assistant Professor an der Stanford University.
Seine Forschungsinteressen liegen insbesondere im Bereich von Künstlicher Intelligenz, High-Performance Computing und Simulation.

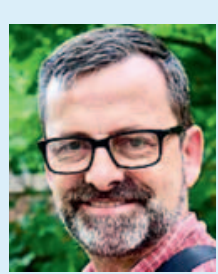

Prof. Philippe Lambin

ist Direktor des Departments für Radioonkologie an der Maastricht University in den Niederlanden. Sein Forschungsschwerpunkt ist die Onkologie mit speziellem Fokus auf der Anwendung von multiparametrischer Bildgebung und Radiomics.

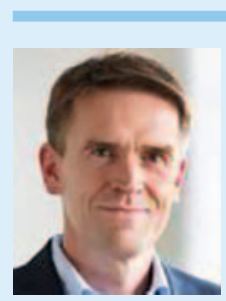

Prof. Wolfgang Wick

ist Direktor der Klinik für Neurologie am Universitätsklinikum Heidelberg. Sein Schwerpunkt ist die Behandlung von Hirntumoren. Als einer der führenden Wissenschaftler in Europa ist er Mitglied in vielen deutschsprachigen und europäischen Arbeitsgemeinschaften im Bereich Neuroonkologie. Herr Wick hat unter anderem 2015 den Deutschen Krebspreis erhalten.

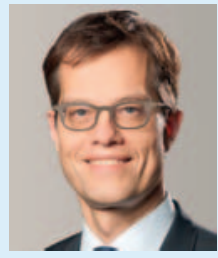

Prof. Martin Bendszus

ist Direktor der Abteilung für Neuroradiologie am Universitätsklinikum Heidelberg. Aus der Heidelberger Klinik stammen zahlreiche viel beachtete Arbeiten zur Bildgebung von Hirntumoren.

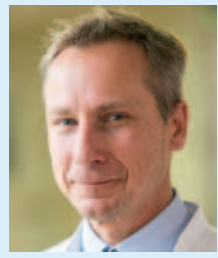

Prof. Bernhard Iglseder ist Vorstand der Universitätsklinik für Geriatrie an de Christian-Doppler-Klinik in Salzburg. Sein klinischer und auch wissenschaftlicher Forschungsschwerpunkt sind dementielle Erkrankungen.

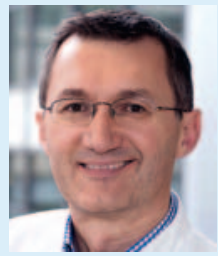

\section{PD Dr. Karl Egger}

ist Oberarzt in der Klinik für Neuroradiologie des Universitätsklinikums Freiburg. In jüngster Zeit hat er sich intensiv mit der Anwendung von Künstlicher Intelligenz im Kontext neurodegenerativer Erkrankungen beschäftigt. 


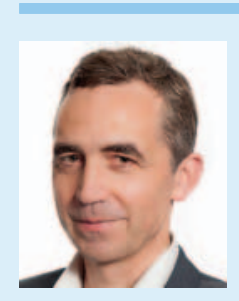

Prof. Bernhard Hemmer

ist Direktor der Klinik für Neurologie am Klinikum rechts der Isar der Technischen Universität München. Er zählt zu den führenden Neurologen auf dem Gebiet der Multiplen Sklerose. Er ist Vorstandsmitglied in verschiedenen Forschungsverbünden zur Multiplen Sklerose und aktuell Präsident des „European Committee for Treatment and Research In Multiple Sclerosis (ECTRIMS)“.

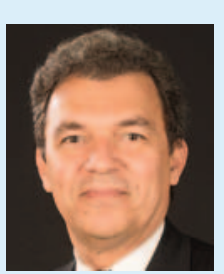

Prof. Tarek Yousry

ist Professor für Neuroradiologie am UCL Institute of Neurology in London. Er ist Mitglied der europäischen MAGNIMS-Gruppe, die sich der Modifizierung und Vereinfachung der MRT-Kriterien für die Diagnose von Multipler Sklerose gewidmet hat.

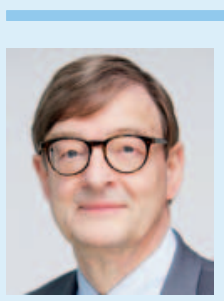

Prof. Otmar Wiestler

ist amtierender Präsident der Helmholtz-Gemeinschaft Deutscher Forschungszentren. Als Neuropathologe war er wesentlich an der Weiterentwicklung der WHO-Klassifizierung der

Hirntumoren beteiligt. Vor seiner Tätigkeit in der Helmholtz-Gemeinschaft war er von 2004 bis 2015 Vorsitzender und wissenschaftliches Mitglied des Stiftungsvorstands des Deutschen Krebsforschungszentrums (DKFZ) in Heidelberg.

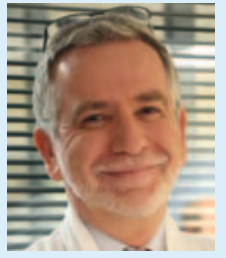

Prof. Georges Rodesch

ist Direktor des Departments für Neuroradiologie des Hopital Foch in Paris. Sein klinischer und wissenschaftlicher Forschungsschwerpunkt ist spinale Gefäßanatomie und interventionelle Versorgung spinaler Gefäßmissbildungen.

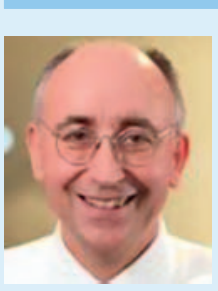

Prof. Axel Stäbler

ist niedergelassener Radiologe in München und hat seinen Schwerpunkt im Bereich der muskuloskelettalen Radiologie. Er ist ein viel beachteter und didaktisch äußerst versierter Redner

auf vielen nationalen und internationalen Fachkongressen.

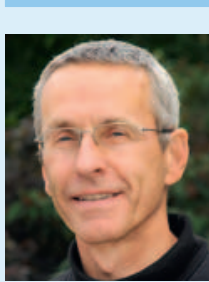

\section{Prof. Ulrich Dirnagl}

ist Direktor der Abteilung für Experimentelle Neurologie an der Charité in Berlin. Seine Forschungsschwerpunkte liegen im Bereich der Regulation des zerebralen Blutflusses und der Pathophysiologie des Schlaganfalls.

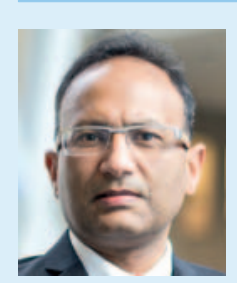

\section{Prof. Mayank Goyal}

ist Professor für Radiologie und klinische Neurowissenschaften an der University von Calgary in Kanada. Sein klinischer und wissenschaftlicher Schwerpunkt ist die interventionelle Versorgung von Schlaganfällen. Prof. Goyal war u. a. Principal Investigator der zwei großen Schlaganfallstudien ESCAPE und SWIFT PRIME.

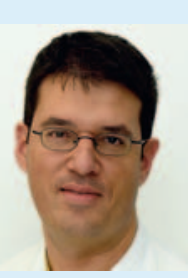

Prof. Nikolaos Koutsouleris

ist Psychiater und beschäftigt sich mit multiparametrischen Mustererkennungsverfahren um HochrisikoPersonen eine genaue Einzelfallvorhersage zu psychotischen Erkrankungen zu er-

möglichen. Er ist Initiator der internationalen Früherkennungsstudie PRONIA (Personalised Prognostic Tools for Early Psychosis Management).

\section{Power Pitch-Vorträge -} Spannende Forschung in höchster Konzentration

Über 200 wissenschaftliche Abstracts wurden für den neuroRAD 2019 eingereicht, darunter eine hohe Anzahl hervorragender Beiträge zum aktuellen Stand der Forschung in der Neuroradiologie. Um diesen herausragenden Beiträgen Raum im Programm zu geben, finden erstmals „PowerPitch-Vorträge“ statt. In maximal zwei Minuten Präsentationszeit präsentieren junge Kolleginnen und Kollegen hier ein Schlaglicht der Wissenschaft nach dem anderen vor einem großen Publikum. Seien Sie gespannt! Die Power-Pitches finden an von Donnerstag bis Samstag statt:

\section{Donnerstag, 10. Oktober 2019}

8:15-10:15: Computational Neuroimaging: Künstliche Intelligenz, Machine Learning, Big Data

11:15-13:15: MS Neues aus Therapie und Diagnostik

16:00-18:00: Zerebrovaskuläre Erkrankungen

\section{Freitag, 11. Oktober 2019}

08:30-10:30: Neuroonkologie

15:30-17:30: Wirbelsäule

\section{Samstag, 12. Oktober 2019}

08:30-10:15: Degenerative ZNS Erkrankungen

\section{NEUROANATOMIE-KURS}

Nach einem überaus erfolgreichen Start in 2018, bieten wir den Neuroanatomie-Kurs in diesem Jahr erneut an. Nutzen Sie den praktischen Kurs zur Oberflächenanatomie des Gehirns an menschlichen Präparaten und sichern Sie sich schnell einen der begehrten Plätze. 


\section{Grundkurs Künstliche Intelligenz in der Neuroradiologie}

Die Begriffe „Künstliche Intelligenz“ und „Big Data“ bestimmen die aktuellen Diskussionen über die Zukunft des Gesundheitswesens im Allgemeinen und der Radiologie/Neuroradiologie im Speziellen.

Bei allen Ungewissheiten, die diesbezüglich bestehen, bietet die Thematik großes Potential für die Verbesserung der Patientenversorgung. Sie ermöglicht insbesondere der radiologischen Gemeinschaft, eine Vorreiterrolle bei der Anwendung entsprechender Techniken einzunehmen.

Heutzutage ist es unerlässlich, ein Grundverständnis für Prinzipien des maschinellen Lernens und resultierende computergestützte Entscheidungssysteme zu entwickeln, um diese künftig erfolgreich im klinischen Betrieb einzusetzen.

Aus diesem Grund bieten wir auf dem neuroRAD 2019 erstmals den „Grundkurs Künstliche Intelligenz in der Neuroradiologie“ an.

Renommierte nationale und internationale Spezialisten vermitteln Ihnen ein Grundverständnis für die Prinzipien des maschinellen Lernens - und dies praxistauglich und anhand klarer Lernziele. Vorkenntnisse sind nicht erforderlich!

\section{Sichern Sie sich jetzt einen der wenigen Plätze und seien Sie am Puls der Zeit!}

Das Programm finden Sie unter neurorad.de $>$ Programm.

Die Anmeldung finden Sie unter neurorad.de $>$ Anmeldung.
Premiere auf dem

neuroRAD 2019:

Die „StAR-Lounge“

Die StAR-Lounge ("Start-up and Applied Research") feiert Premiere auf dem neuroRAD 2019. Hier präsentieren über 20 Startups, Forschungsgruppen und Anwender ihre Lösungen und Ideen zu Themen wie Künstliche Intelligenz, Machine Learning, Datenmanagement, Robotics und anderen Themen der Zukunft.

Darüber hinaus erwarten Sie in spannenden Podiumsdiskussionen aktuelle Themen der Zeit:

- Neuroradiologen bei der Agentur für Arbeit - macht KI uns überflüssig?

- Die Zukunft jetzt! - Wie integrieren wir innovative Konzepte in die Patientenversorgung?

In unserer Youtube-Reihe „Start-up Shuttle“ präsentieren wir Ihnen einige der Akteure. Im ersten Video stellt sich apoQlar vor ein Start-up, das Mixed und Augmented Reality in der Radiologie schon heute einsetzt. In weiteren Videos lernen Sie u. a. die Start-ups deepc und BIOMAX kennen.

Alle Informationen zur StAR-Lounge und zum Start-up Shuttle finden Sie unter neurorad.de $>$ StAR-Lounge.

\section{Weiterbildung/Fit für den Facharzt}

Es erwartet Sie auch dieses Jahr wieder ein umfangreiches Weiterbildungsangebot quer durch die gesamte Neuroradiologie, präsentiert von ausgewiesenen Rednern. U.a.: „Schlaganfälle bei Kindern“, „Neuropathologisch-neuroradiologische Korrelationen“, „Wie lerne ich Interventionen?“, „Bildgebung beim Shaken-Baby-Syndrom“, „Irrtümer in der Neuroradiologie“, „Crashkurs Notfallbildgebung“, „Bildgebung der Autoimmunenzephalitiden und Vaskulitiden“, „Traumatologie und Entzündungen der Wirbelsäule“ und vieles mehr.

\section{Pepper zu Gast auf dem}

\section{neuroRAD 2019}

Erneut wird mit dem MTRA-Schülertag auch dem radiologischen Nachwuchs die Möglichkeit geboten in die Welt der Neuroradiologie einzutauchen.

\section{Der MTRA-Schülertag 2019 findet am Donnerstag, 10. Oktober von $9 \mathrm{Uhr}-17$ Uhr statt und bietet den Teilnehmern viel- fältige Gelegenheit, die MTRA-Arbeit in der Neuroradiologie kennenzulernen!}

In den ausgedehnten Pausenzeiten können zudem die Key Note-Lectures besucht und natürlich kräftig Kongressluft geschnuppert werden. Das Programm und nähere Informationen finden Sie auf www.neurorad.de

Wir freuen uns über rege Beteiligung der MTRA-Schulen. Selbstverständlich ist dieses Angebot für Schulklassen und zwei begleitende Lehr-MTRA kostenfrei.

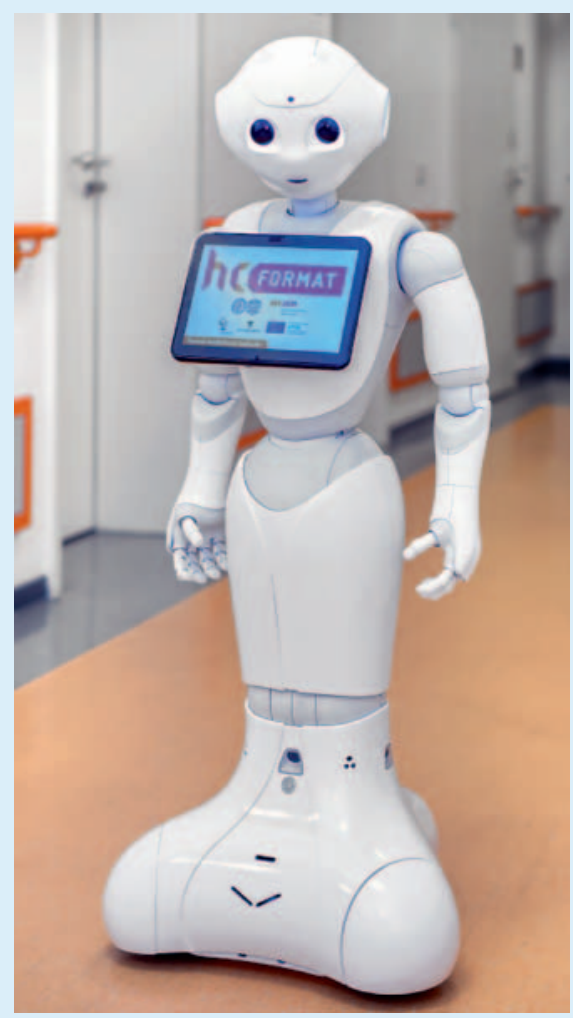

Copyright: Medizinische Fakultät der MartinLuther-Universität Halle-Wittenberg) 


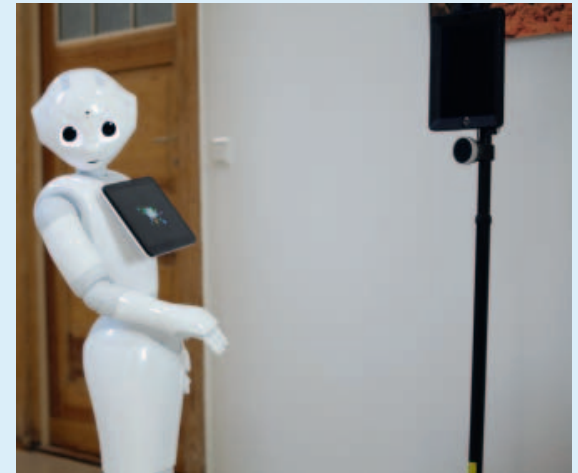

Copyright: Medizinische Fakultät der MartinLuther-Universität Halle-Wittenberg)
Ein ganz besonderer Gast im MTRA-Schülerprogramm: Pepper!

Die Schülerinnen und Schüler können sich auch auf einen ganz besonderen Gast freuen: Pepper, der Pflegeroboter der Uni Halle kommt zum neuroRAD 2019 nach Frankfurt.

Pepper soll Pflegerinnen und Pfleger bei ihrer Arbeit unterstützen. Mit seinen großen Augen, seinem Lächeln und einer piepsigen Stimme soll er zudem Patientinnen und Patienten die Angst vor Untersuchungen oder Therapien nehmen.

\section{DIE KONGRESSDATEN:}

Thema „Robotik in der Radiologie Unterstützung oder Gefahr?“

Do., 10.10.2019, 08:45-09:30 Uhr im MTRA-Schülerprogramm

Alle Informationen zur Jahrestagung, zur Anmeldung, zum Programm und den Workshops finden Sie auf neurorad.de

Bitte melden Sie Ihre Klassen über die Online-Registrierung auf www.neurorad.de an. 